\title{
Cloud-Chamber Study of the Production and Decay of Strange Particles*
}

\author{
G. H. Trilling $\dagger$ and R. B. LE'ighton \\ California Institute of Technology, Pasadena, California
}

(Received September 4, 1956)

\begin{abstract}
Out of 1242 decay events observed in a magnetic cloud chamber array triggered on penetrating showers, a total of 54 associated $V$-particle pairs has been obtained. All the associations observed are consistent with the "strangeness" selection rules. A statistical analysis of the numbers of double and single events has been used to obtain rough estimates of the fractions of neutral $K$ particles and neutral hyperons which decay by "invisible" modes, and of the relative frequencies of occurrence of various production processes. The main uncertainties in the resulting values are discussed in detail.
\end{abstract}

\section{INTRODUCTION}

$\mathrm{T}$ HE production of $V$ particles in pairs was suggested $^{1}$ very early as a possible way of reconciling their copious production with their long lifetime. More recently the idea of pair production has been replaced by that of associated production in which only certain combinations of these particles may be produced together. Various schemes have been proposed to classify $V$ particles according to their production associations. ${ }^{2}$ One of the most useful of these is the socalled "strangeness number" classification introduced by Gell-Mann and Pais. Under this scheme all strongly interacting particles are assigned a new quantum number called the strangeness, denoted by the symbol $S$, which can assume only integral values. The strangeness numbers assigned to the presently known or postulated particles are shown in Table I. According to the rules proposed by Gell-Mann and Pais, the permitted associations of $V$ particles are described by the selection rule that the total strangeness does not change at production, whereas the observed slow decay of $V$ particles is governed by the rule that the strangeness changes by one unit. The consequences of these rules insofar as production is concerned will be discussed in Sec. II.

Early experimental attempts to test the pair production hypothesis by comparing the relative numbers of double and single events in cosmic-ray cloud-chamber photographs seemed to give negative results. ${ }^{3}$ However, these investigations were subject to the important objection that no account was taken of either the possible existence of decay modes having solely neutral secondaries or of $V$ particles of such lifetime that their detection efficiency in a cloud chamber is very small. Indeed, it is now well known that the long lifetime of

\footnotetext{
* Assisted by the joint program of the Office of Naval Research and U. S. Atomic Energy Commission.

$\dagger$ Now at the Ecole Polytechnique, Paris, France.

1 Nambu, Nishijima, and Yamaguchi, Progr. Theoret. Phys. Japan 6, 615 (1951); 6, 619 (1951); A. Pais, Phys. Rev. 86, 663 (1952).

${ }_{2}$ M. Gell-Mann and A. Paris, Proceedings of the 1954 Glasgow Conference on Nuclear and Meson Physics (Pergamon Press, London, 1955); A. Salam and J. C. Polkinghorne, Nuovo cimento 2, 685 (1955).

${ }^{3}$ Leighton, Wanlass, and Anderson, Phys. Rev. 89, 148 (1953);

Fretter, May, and Nakada, Phys. Rev. 89, 168 (1953).
}

$K^{+}$particles $^{4}$ and the short lifetime of $\Sigma^{+}$particles ${ }^{5}$ gives these a relatively poor detection efficiency in ordinary cloud chambers. There is also evidence for the existence of neutral modes of decay, ${ }^{6}$ so that the results of the early experimental work on pair production of $V$ particles must be considered inconclusive.

The work of Shutt and his collaborators at Brookhaven $^{7}$ gave the first positive experimental evidence in favor of associated production, and further work at the Cosmotron has continued to strengthen this evidence. ${ }^{8}$ In addition, other cosmic-ray research both with cloud chambers and photographic emulsions has given results in agreement with the hypothesis of associated production. ${ }^{9-11}$ Finally, it should be noted that recent photographic emulsion work on the interactions of $K^{+}$ and $K^{-}$mesons has given additional strong support to the predictions of the strangeness selection rules. ${ }^{12}$

The results to be described in the present paper were obtained by using data from the 48-in. magnet cloud chambers. ${ }^{13}$ This work consisted of two parts: first, a detailed study of all associated $V$-particle events to

TABLE I. Strangeness classification.

\begin{tabular}{rl}
\hline \hline$s$ & \multicolumn{1}{c}{ Particles } \\
\hline 1 & $K^{+}, K^{0}$ \\
0 & $p, n, \pi^{ \pm}, \pi^{0}$ \\
-1 & $\Lambda^{0}, \Sigma^{0}, \Sigma^{ \pm}, \bar{K}^{0}, K^{-}$ \\
-2 & $\Xi^{-}, \Xi^{0}$ \\
\hline \hline
\end{tabular}

${ }^{4}$ V. Fitch and R. Motley, Phys. Rev. 101, 496 (1956); Alvarez, Crawford, Good, and Stevenson, Phys. Rev. 101, 503 (1956).

${ }^{5}$ Fry, Schneps, Snow, and Swami, Phys. Rev. 100, 950 (1955)

${ }^{6}$ Asher, Moyer, and Parker, Bull. Am. Phys. Soc. Ser. II, 1, 184 (1956).

${ }^{7}$ Fowler, Shutt, Thorndike, and Whittemore, Phys. Rev. 91, 1287 (1953); 93, 861 (1954); 98, 121 (1955).

${ }^{8}$ W. D. Walker, Phys. Rev. 98, 1407 (1955); Block, Harth, Fowler, Shutt, Thorndike, and Whittemore, Phys. Rev. 99, 261 (1955); W. D. Walker and W. D. Shepard, Phys. Rev. 101, 1810 (1956).

${ }^{9}$ Thomspon, Burwell, Hugget, and Karzmark, Phys. Rev. 95, 1576 (1954).

${ }_{10}$ Sorrels, Leighton, and Anderson, Phys. Rev. 100, 1457 (1955).

11 Dahanayake, Francois, Fujimoto, Iredale, Waddington, and Yasin, Phil. Mag. 45, 855 (1954).

12 Lannuti, Chupp, Goldhaber, Goldhaber, Helmy, Iloff, Pevsner, and Ritson, Phys. Rev. 101, 1617 (1956).

${ }_{13}$ G. H. Trilling and R. B. Leighton, Phys. Rev. 100, 1468 (1955). 
test whether the predictions of the strangeness scheme are fulfilled; and second, a statistical study of single and double events to obtain information on neutral decay modes and on relative production probabilities for various associations of particles.

\section{PRINCIPAL PRODUCTION PROCESSES FOR STRANGE PARTICLES}

\section{A. Theory}

The strangeness selection rules require than an $S=-1$ particle be produced in association with an $S=+1$ particle, and than an $S=-2$ particle be produced with any two $S=+1$ particles. According to Table I, this means that $\Lambda^{0}, \Sigma^{0}, \Sigma^{ \pm}, \bar{K}^{0}$ or $K^{-}$must be produced with $K^{0}$ or $K^{+}$, and that $\Xi^{-}$or $\Xi^{0}$ must be produced with $2 K^{0}, 2 K^{+}$, or $K^{0}+K^{+}$.

In connection with these permissible associations the following points should be noted:

1. The $\Xi^{0}$ particle, postulated to decay according to the scheme

$$
\Xi^{0} \rightarrow \Lambda^{0}+\pi^{0}
$$

whose existence is predicted by the strangeness theory, has as yet not been found experimentally.

2. The existence of the $\Sigma^{0}$, which is supposed to decay rapidly $\left(\sim 10^{-20} \mathrm{sec}\right)$ according to the scheme

$$
\Sigma^{0} \rightarrow \Lambda^{0}+\gamma
$$

is not firmly established, though there is considerable evidence in its favor. ${ }^{7,8}$ It is difficult to distinguish experimentally between the direct production of a $\Lambda^{0}$ and the production of a $\Sigma^{0}$ which subsequently decays into a $\Lambda^{0}$.

3. Experimentally, one observes several different decay schemes for $K^{+}$particles, all of which seem to correspond to about the same mass and lifetime. However, there are theoretical reasons for believing that not all of these schemes correspond to the same physical particle. Arguments on the basis of spin and parity ${ }^{14}$ suggest that there may be at least two different types of $K^{+}$particles, one being responsible for all $\tau^{+}$decays and the other giving all $\theta^{+}$decays.

4. According to the strangeness theory, every $K^{+}$ particle must have a neutral counterpart. At present, only the existence of the neutral counterpart $\left(\theta^{0}\right)$ of the $\theta^{+}$has been firmly established, but several cases of socalled "anomalous $\theta^{0}$ " events have been observed, ${ }^{15}$ some of which might be examples of the expected $\tau^{0}$ decay.

5. Gell-Mann and Pais have suggested ${ }^{16}$ from theoretical arguments that the $\theta^{0}$ particles should manifest themselves experimentally as two different particles, which they call $\theta_{1}{ }^{0}$ and $\theta_{2}{ }^{0}$, produced in equal numbers,

14 R. Dalitz, Phys. Rev. 94, 1046 (1954); Orear, Harris, and Taylor, Phys. Rev. 102, 1676 (1956).

${ }_{15}$ Van Lint, Anderson, Cowan, Leighton, and York, Phys. Rev. 94, 1732 (1954).

${ }_{16}$ M. Gell-Mann and A. Pais, Phys. Rev. 97, 1387 (1955). having equal mass, spin and parity, but with markedly different lifetimes, only one of the particles $\left(\theta_{1}{ }^{0}\right)$ being able to decay into two $\pi$-meson secondaries. By the same argument, $\bar{\theta}^{0}$ particles should exhibit themselves as half $\theta_{1}{ }^{0}$ and half $\theta_{2}{ }^{0}$. These authors suggest that the lifetime of the $\theta_{2}{ }^{0}$ may be such as to make the detection of its decay in a cloud chamber improbable. Some of the anomalous $\theta^{0}$ events mentioned above might be examples of these predicted $\theta_{2}{ }^{0}$ decays.

\section{B. Experiment}

The data to be discussed in this paper were obtained from approximately 50000 photographs taken with the 48 -in. cloud chambers. The various $V$-particle decays were classified according to the accepted decay schemes using the methods outlined in Table II. The following points should be noted in connection with Table II.

1. Only one of the three methods given for the identification of a $\theta^{0}$ decay requires that the positive

TABLE II. Identification procedures used in the classification of events. ( $P, I, M=$ momentum, ionization, and mass of primary, respectively; $P^{ \pm}, I^{ \pm}, M^{ \pm}=$momentum, ionization, and mass of positive or negative secondary; $\theta=$ opening angle of $V^{\theta}$ decay;

\begin{tabular}{|c|c|c|}
\hline Particle & Necessary information & Condition for identification \\
\hline $\begin{array}{l}\Lambda^{0} \\
\theta^{0} \\
\theta^{0} \\
\theta^{0} \\
K_{L^{ \pm b}} \\
\tau^{ \pm} \\
\Xi^{-} \\
\text {" } \Lambda^{0} " \mathrm{c} \\
\text { " } K_{L}{ }_{L}^{+"}\end{array}$ & $\begin{array}{l}P^{+}, I^{+} \\
P^{+}, I^{+} \\
P^{-}, \theta \\
\text { Sign of } P^{+-} P^{-} \\
P, I \quad \\
\quad \cdots \\
\quad \cdots \\
P^{-}, \theta\end{array}$ & $\begin{array}{l}273 m_{e}<M^{+} \sim 1840 m_{e} \\
M^{+}<1840 m_{e} \\
P^{-} \sin \theta>118 \mathrm{Mev} / c \\
P^{+}-P^{-}<0^{\mathrm{a}} \\
M<\sim 2000 m_{e} \\
3 \text { charged secondaries } \\
V^{0} \operatorname{secondary} \\
P^{-} \sin \theta \leqslant 118 \mathrm{Mev} / c \\
t>5 \times 10^{-10} \mathrm{sec}\end{array}$ \\
\hline
\end{tabular}
$t=$ time of flight of primary inside the chamber.)

a It is taken for granted that very slow $\Lambda^{0^{\prime}}$ s which may satisfy this criterion will be identified from $P^{+}$and $I^{+}$

b The term $K_{L}$ includes only $K$ mesons which do not decay by the $\tau$ mode. - The " $\Lambda$ " " and " $K_{L}$ " indentifications are given only to events not otherwise identifiable.

secondary be lighter than a proton. The other two methods are based on the dynamics of $\Lambda^{0}$ and $\theta^{0}$ decay, and depend on the fact that the $Q$ value of the known $\Lambda^{0}$ decay is $37 \mathrm{Mev}$. If particles having a proton secondary and a $Q$ value significantly greater than $37 \mathrm{Mev}$ were present, these would sometimes be classified as $\theta^{0}$ 's. If such decays are present, however, their proportions are exceedingly small.

2. The " $\Lambda$ " identification is based on the fact that less than $10 \%$ of ordinary $\theta^{0}$ events can fulfill the given criterion, ${ }^{17}$ while all $\Lambda^{0}$ events must fulfill it. Thus, whereas not every event labeled " $\Lambda^{0}$ " is in fact a $\Lambda^{0}$, all but a few of these events are $\Lambda^{0}$ s.

3. The " $K^{+}$" identification depends upon the fact that the lifetime of the $\Sigma^{+}$is about $5 \times 10^{-11} \mathrm{sec} .{ }^{5}$ Hence events with time of flight greater than $5 \times 10^{-10} \mathrm{sec}$ are almost certainly not $\Sigma^{+}$, and are hence assumed to be $K^{+}$.

4. A detailed examination of a large sample of single

${ }^{17}$ J. P. Astbury, Nuovo cimento 12, 387 (1954). 
neutral events, to be discussed in Sec. III, has shown that with the $\Lambda^{0}$ and $\theta^{0}$ criteria given in Table II one can positively identify about $40 \%$ of the $\Lambda^{0}$ 's and $55 \%$ of the $\theta^{0}$ 's. On the other hand, if one lumps the " $\Lambda^{0 "}$ 's and $\Lambda^{0}$ 's together, then the identification efficiency for $\Lambda^{0}$ 's goes up to about $85 \%$ accompanied, however, by a slight contamination of $\theta^{0}$ s.

Table III gives a breakdown on the events obtained up to the present time. From this sample a total of 54 cases were found which showed the decays of two or more associated $V$ particles. The term "associated" is intended to convey the fact that the lines of flight of the primaries of these decays intersect, within the errors of measurement, in the producing material surrounding the chambers. Although this strongly suggests that the particles were produced in the same interaction, it must be noted that there is no experimental way of distinguishing whether the two members of the "associated pair" came from a single interaction or from two different interactions, perhaps in the same nucleus.

Table IV lists the identifications of the members of the 36 pairs of associated neutral $V$ particles obtained.

TABLE III. Breakdown of the decay events obtained.

\begin{tabular}{lrlr}
\hline \hline \multicolumn{2}{c}{ Identified events } & \multicolumn{2}{c}{ Unidentified events } \\
\hline$\Lambda^{\theta \text { a }}$ & 228 & $V^{0}$ & 564 \\
$\theta^{0}$ & 238 & $V^{+}$ & 48 \\
$K_{L^{+}}$ & 42 & $V^{-}$ & 93 \\
$K_{L^{-}}$ & 7 & $V^{ \pm}$ & 10 \\
$\Xi^{-}$ & 6 & & \\
$\tau^{ \pm}$ & 6 & & \\
\hline
\end{tabular}

a These do not include " $\Lambda^{0 "}$ " and " $K_{L}{ }^{+}$" events. The latter are included in the $V^{0}$ and $V^{+}$categories, respectively.

Inspection of this table leads one to the following observations :

1. Every event in which both members of the pair are completely identified is a $\Lambda^{0}-\theta^{0}$ pair. There are no identified $\Lambda^{0}-\Lambda^{0}$ or $\theta^{0}-\theta^{0}$ pairs.

2. If the " $\Lambda^{0}$ " events are lumped with the $\Lambda^{0}$ events, thus permitting an identification of $\Lambda^{0}$ 's which is not nearly as energy sensitive as the requirement that the positive secondary be identified as a proton, then three $\Lambda^{0}-\Lambda^{0}$ pairs are obtained. However, one would expect, if all events were $\Lambda^{0}-\theta^{0}$ pairs that about three $\theta^{0}$ decays would be identified as " $\Lambda^{0}$ " events. In fact, just three $\theta^{0}$ decays satisfying the symmetrical requirement $P^{+} \sin \theta<118 \mathrm{Mev} / c$ are present in the sample. Hence, there is no evidence for even a single $\Lambda^{0}-\Lambda^{0}$ pair. This statement is particularly strong in view of the $85 \%$ identification probability for $\Lambda^{0}$ s, when " $\Lambda^{0 "}$ " and $\Lambda^{0}$ events are lumped together.

3. Other evidence for the absence of $\theta^{0}-\theta^{0}$ pairs among the events under discussion can be obtained from a consideration of the number of $\theta^{0}-V^{0}$ events. From the identification probabilities given above, one calculates that about $8 \%$ of $\Lambda^{0}-\theta^{0}$ events should be
TABLE IV. Identities of the members of the $36 V^{0}-V^{0}$ pairs.

\begin{tabular}{lrrrr}
\hline \hline & $\Lambda^{0}$ & $\theta^{0}$ & " $\Lambda^{0 "}$ & $V^{0}$ \\
\hline$\Lambda^{0}$ & 0 & & & \\
$\theta^{0}$ & 10 & 0 & & \\
$\Lambda^{0}{ }^{0}$ & 2 & 5 & 1 & 2 \\
\hline \hline
\end{tabular}

identified as $V^{0}-\theta^{0}$ pairs while $50 \%$ of the $\theta^{0}-\theta^{0}$ pairs should be so identified. The fact that only $4 V^{0}-\theta^{0}$ events were obtained from 36 associated pairs strongly suggests that the proportion of $\theta^{0}-\theta^{0}$ cases is small.

4. The numbers of identified events in Table IV are completely consistent with the assumption that all pairs consist of a $\Lambda^{0}$ and a $\theta^{0}$ and that the identification probabilities are those given above.

The net conclusion of these observations is that essentially all of the 36 neutral pairs observed are $\Lambda^{0}-\theta^{0}$ pairs. This is in good agreement with the strangeness scheme which forbids $\Lambda^{0}-\Lambda^{0}$ associations and inhibits $\theta^{0}-\theta^{0}$ production because of the higher threshold energy required. These results also indicate that plural production contributes very little to the pair events observed.

The identifications of the members of 15 associated $V^{0}-V^{ \pm}$pairs, excluding $\Xi^{-}$events, are shown in Table $\mathrm{V}$. Here the following observations apply:

1. There are no identified $\Lambda^{0}-V^{-}$events present, in complete agreement with the strangeness selection rules.

2. The principal associations seem to be $\Lambda^{0}-K^{+}$and $\theta^{0}-V^{-}$. The latter are probably $\theta^{0}-\Sigma^{-}$pairs $\left(\theta^{0}-\Sigma^{+}\right.$ pairs are more difficult to detect because of the shorter lifetime of the $\Sigma^{+}$).

3. All observed associations are completely compatible with the strangeness theory.

To these data should be added the observation of a $\Xi^{-}-\theta^{0}-\theta^{0}{ }^{10}$ a $\Xi^{-}-\theta^{0}$, and a $K^{+}-V^{-}$. The first of these is in spectacular agreement with the strangeness selection rules, and the others are also in good agreement. All of these results serve to confirm, on the one hand, the validity of the strangeness rules, and on the other hand the fact that all of the pair events observed are probably cases of true associated production.

\section{STATISTICAL ANALYSIS OF THE DATA}

\section{A. Theory}

In view of the results just given, it seems reasonable to assume that the processes responsible for the large majority of both single and double events are those in which one hyperon and one $K$ meson are produced in

TABLE V. Identities of the members of the $15 V^{0}-V^{ \pm}$pairs.

\begin{tabular}{lcccc}
\hline \hline & $\Lambda^{0}$ & $\theta^{0}$ & " $\Lambda^{0^{\prime}}$ & $V^{0}$ \\
\hline$K_{L^{+}}$ & 1 & 1 & 0 & 0 \\
" $K_{L^{+}}{ }^{+}$ & 2 & 0 & 1 & 1 \\
$V^{+}$ & 1 & 1 & 0 & 0 \\
$V^{-}$ & 0 & 4 & 0 & 1 \\
$V^{ \pm}$ & 0 & 1 & 0 & 1 \\
\hline
\end{tabular}


association, i.e., in which the observed pairs are $\Lambda^{0}-K^{0}$, $\Lambda^{0}-K^{+}, \Sigma^{ \pm}-K^{0}$, and $\Sigma^{ \pm}-K^{+}$. ( $\Lambda^{0}$ events will of course include both those directly produced and those resulting from the decays of $\Sigma^{0}$ 's.) With this assumption, one can derive considerable information concerning the production and decay of $V$ particles from studies of the numbers and types of single and double decay events.

Let $\rho_{\theta}$ be that fraction of all neutral $K$ particles which decay by the mode,

$$
\theta^{0} \rightarrow \pi^{+}+\pi^{-}+214 \mathrm{Mev}, \quad \tau \approx 10^{-10} \mathrm{sec} .
$$

Similarly, let $\rho_{\Lambda}$ be that fraction of $\Lambda^{0}$ particles which decay by the mode,

$$
\Lambda^{0} \rightarrow p+\pi^{-}+37 \mathrm{Mev}, \quad \tau=3.6 \times 10^{-10} \mathrm{sec} .
$$

Neutral particles which do not decay in these ways and which will be said to decay by "invisible modes" may be in one or both of the following categories: (1) those whose secondaries are all neutral; (2) those which decay into charged secondaries with a lifetime of such magnitude as to make their detection probability in the chamber negligible.

Define $P\left(\Lambda^{0} K^{0}\right)$ to be the number of $\Lambda^{0}-K^{0}$ pairs actually created ${ }^{18}$ in the production layer of a cloud chamber during a specified time of operation. Let $N\left(\Lambda^{0} \theta^{0}\right)$ be the number of these pairs actually observed in the chamber as $\Lambda^{0}-\theta^{0}$ decays. Similarly define the quantities $P\left(\Lambda^{0} K^{+}\right), P\left(\Sigma^{ \pm} K^{0}\right), P\left(\Sigma^{ \pm} K^{+}\right), \quad N\left(\Lambda^{0} K^{+}\right)$, $N\left(\Sigma^{ \pm} \theta^{0}\right)$, and $N\left(\Sigma^{ \pm} K^{+}\right)$. Let $N\left(\Lambda^{0}\right), N\left(\theta^{0}\right), N\left(\Sigma^{ \pm}\right)$, and $N\left(K^{+}\right)$be, respectively, the observed numbers of $\Lambda^{0}, \theta^{0}, \Sigma^{ \pm}$, and $K^{+}$decays, either singly or in association with another particle, during the same period of operation. Finally, let the quantities $\pi\left(\Lambda^{0} \theta^{0}\right), \pi\left(\Lambda^{0} K^{+}\right)$, $\pi\left(\Sigma^{ \pm} \theta^{0}\right), \pi\left(\Sigma^{ \pm} K^{+}\right), \pi\left(\Lambda^{0}\right), \pi\left(\theta^{0}\right), \pi\left(K^{+}\right)$, and $\pi\left(\Sigma^{ \pm}\right)$ represent the probabilities that, if the events referred to decay visibly in the sense of the definition previously given, they will be observed in the chamber. Let a bar over any of these symbols denote an average over the events actually created and angle brackets $(\langle\rangle)$ denote an average over the events observed.

The following relations will then apply:

$$
\begin{aligned}
N\left(\Lambda^{0} \theta^{0}\right) & =P\left(\Lambda^{0} K^{0}\right) \rho_{\Lambda} \rho_{\theta} \bar{\pi}\left(\Lambda^{0} \theta^{0}\right), \\
N\left(\Lambda^{0} K^{+}\right) & =P\left(\Lambda^{0} K^{+}\right) \rho_{\Lambda} \bar{\pi}\left(\Lambda^{0} K^{+}\right), \\
N\left(\Sigma^{ \pm} \theta^{0}\right) & =P\left(\Sigma^{ \pm} K^{0}\right) \rho_{\theta} \bar{\pi}\left(\Sigma^{ \pm} \theta^{0}\right), \\
N\left(\Sigma^{ \pm} K^{+}\right) & =P\left(\Sigma^{ \pm} K^{+}\right) \bar{\pi}\left(\Sigma^{ \pm} K^{+}\right), \\
N\left(\Lambda^{0}\right) & =\left[P\left(\Lambda^{0} K^{0}\right)+P\left(\Lambda^{0} K^{+}\right)\right] \rho_{\Lambda} \bar{\pi}\left(\Lambda^{0}\right), \\
N\left(\theta^{0}\right) & =\left[P\left(\Lambda^{0} K^{0}\right)+P\left(\Sigma^{ \pm} K^{0}\right)\right] \rho_{\theta} \bar{\pi}\left(\theta^{0}\right), \\
N\left(K^{+}\right) & =\left[P\left(\Lambda^{0} K^{+}\right)+P\left(\Sigma^{ \pm} K^{+}\right)\right] \bar{\pi}\left(K^{+}\right), \\
N\left(\Sigma^{ \pm}\right) & =\left[P\left(\Sigma^{ \pm} K^{0}\right)+P\left(\Sigma^{ \pm} K^{+}\right)\right] \bar{\pi}\left(\Sigma^{ \pm}\right) .
\end{aligned}
$$

\footnotetext{
${ }^{18}$ It should be clearly understood that the pairs referred to here are those which actually leave the nucleus in which the interaction took place. They are not necessarily the pairs produced in the initial interaction, since secondary interactions in which strangeness is conserved may take place in the same nucleus with appreciable probability.
}

Because of the statistical limitations of the present experimental data, only Eqs. (1), (5), (6), and (7) can be used effectively for further analysis. Combining (1) with (5), (1) with (6), and (6) with (7), one obtains:

$$
\begin{aligned}
& I_{1} \equiv \frac{N\left(\Lambda^{0}\right)}{\bar{\pi}\left(\Lambda^{0}\right)} \frac{\bar{\pi}\left(\Lambda^{0} \theta^{0}\right)}{N\left(\Lambda^{0} \theta^{0}\right)}=\frac{1}{\rho_{\theta}}\left[1+\frac{P\left(\Lambda^{0} K^{+}\right)}{P\left(\Lambda^{0} K^{0}\right)}\right], \\
& I_{2} \equiv \frac{N\left(\theta^{0}\right)}{\bar{\pi}\left(\theta^{0}\right)} \frac{\bar{\pi}\left(\Lambda^{0} \theta^{0}\right)}{N\left(\Lambda^{0} \theta^{0}\right)}=\frac{1}{\rho_{\Lambda}}\left[1+\frac{P\left(\Sigma^{ \pm} K^{0}\right)}{P\left(\Lambda^{0} K^{0}\right)}\right], \\
& I_{3} \equiv \frac{N\left(K^{+}\right)}{\bar{\pi}\left(K^{+}\right)} \frac{\bar{\pi}\left(\theta^{0}\right)}{N\left(\theta^{0}\right)}=\frac{1}{\rho_{\theta}}\left[\frac{P\left(\Lambda^{0} K^{+}\right)+P\left(\Sigma^{ \pm} K^{+}\right)}{P\left(\Lambda^{0} K^{0}\right)+P\left(\Sigma^{ \pm} K^{0}\right)}\right] .
\end{aligned}
$$

The quantities $I_{1}, I_{2}$, and $I_{3}$ are combinations which can be experimentally determined, and from which information concerning nonvisible modes of decay and the various modes of production can be obtained.

\section{B. Experimental Evaluation of $I_{1}, I_{2}$, and $I_{3}$}

\section{Calculation of $I_{1}$ and $I_{2}$}

The main problem involved in measuring the quantities $I_{1}$ and $I_{2}$ is in the evaluation of the $\bar{\pi}$ 's. The lifetimes used are the following:

$$
\begin{aligned}
& \tau_{\Lambda}=3.6 \times 10^{-10} \mathrm{sec}, \\
& \tau_{\theta}=1.3 \times 10^{-10} \mathrm{sec} .
\end{aligned}
$$

In carrying out the analysis, only events with origins in the $5 \mathrm{~cm}$ of production layer adjacent to the chamber were considered. The probability, $\pi$, of observation of each case was calculated by assuming that the event was drawn from a population of events of the same momenta and emitted in the same direction, but with origins uniformly distributed throughout the volume of production layer considered. The mean probability of observation $\bar{\pi}$, averaged over events produced, was then obtained in terms of the average of $1 / \pi$ over observed events, $\langle 1 / \pi\rangle$, using the relation,

$$
\bar{\pi}=\langle 1 / \pi\rangle^{-1} \text {. }
$$

The main limitation in the accuracy of the results comes from the statistical inaccuracy inherent in the small number of double events. In order to reduce the labor of computation, therefore, only those single events occurring in a certain subregion of the chambers were considered, the numbers being suitably scaled when put into formulas (9a) and (9b).

The validity of the above procedure and of the use of Eq. (11) rests upon the assumption that the observed events are a fairly good representation of the events produced. That is, one assumes that the observation probabilities of the produced sample are well represented by those of the observed sample. A partial justification of this assumption is provided by Fig. 1 which shows a plot of the average detection probability 
for events produced in the first $5 \mathrm{~cm}$ of production layer above the chambers as a function of $L$, the vertical component of their decay distance $\gamma \beta c \tau$. It should be noted from Fig. 1 that the detection probability varies by only a factor of three as the $\Lambda^{0}$ or $\theta^{0}$ momentum varies from about $200 \mathrm{Mev} / c$ to $10 \mathrm{Bev} / c$. Thus only in the unlikely eventuality that a large fraction of the produced events are outside this very wide range is the analysis given here in large error.

However, a possible difficulty arises from the fact that, because some events are emitted at very large angles to the vertical or even backward in the laboratory system, their detection probability in, say, the top chamber, may be very small or indeed zero. Careful studies were made of the angular distributions of $\Lambda^{0}$ s and $\theta^{0}$ s, and corrections were made for these effects.

Another point which deserves mention is the identification of the single and double events as $\Lambda^{0}$ s and $\theta^{0}$ s. It was, of course, necessary to use all $V^{0}$ events decaying within the allowed regions of the chambers and fulfilling the origin requirements regardless of one's ability to identify them. In addition to the rigid and approximate identification criteria previously discussed, association with identified $\Lambda^{0}$ 's and $\theta^{0}$ 's was also used as a criterion, on the assumption that all $V^{0}$ pairs were $\Lambda^{0}-\theta^{0}$ events. With all these methods of identification only a very small number of events remained which gave no preference as to being identified as $\Lambda^{0}$ 's or $\theta^{0}$ 's. These events were apportioned among $\Lambda^{0}$ 's and $\theta^{0}$ 's in the same ratio as the identified events. The momenta of all events were computed by using all information derived from measurements on the secondaries plus knowledge of the dynamics of the decays.

\section{Calculation of $I_{3}$}

Somewhat similar procedures were used to calculate the detection probabilities of $K^{+}$'s and $\theta^{0}$ 's as required for the computation of $I_{3}$. In this analysis all $V^{+}$events were assumed to be the decays of $K^{+}$mesons having a

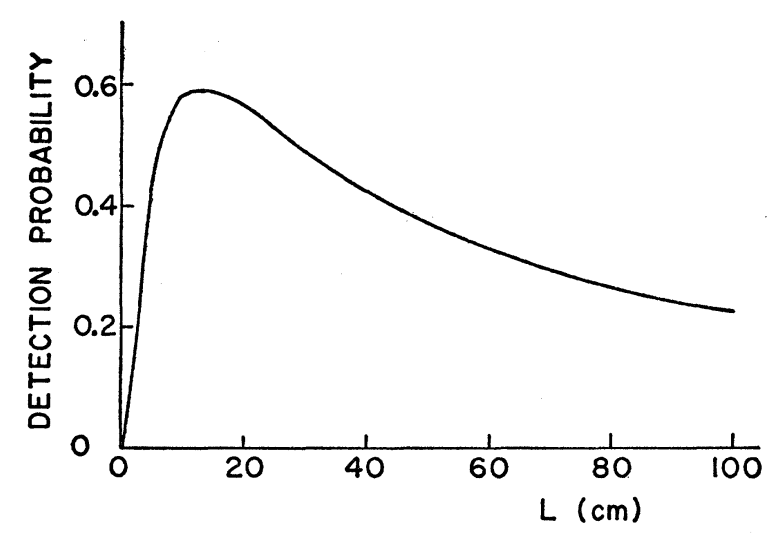

FIG. 1. Average detection probability of downward traveling particles created in the $5 \mathrm{~cm}$ of producing material above each chamber, plotted as a function of their mean decay distance $L=\gamma \beta c \tau$.

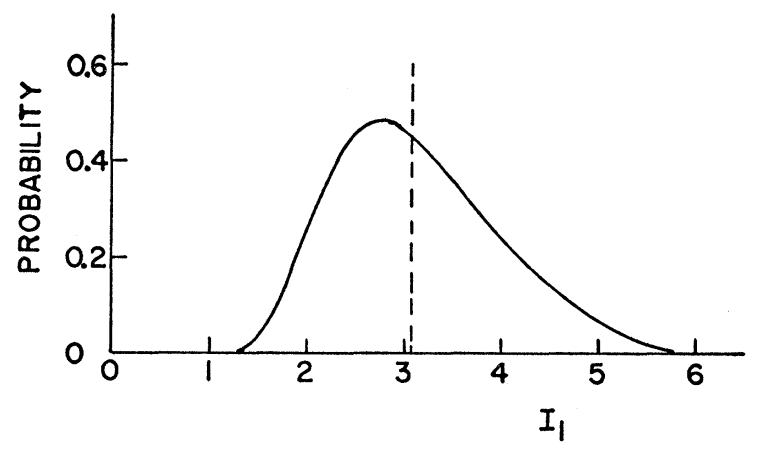

FIG. 2. Expected distribution of $I_{1}$ from a set of measurements of the same statistical accuracy as the present experiment. This curve is computed on the assumption that the true value of $I_{1}$ is the one obtained in the present experiment and shown in the figure by a dashed line.

lifetime $^{4}$

$$
\tau_{K}=1.2 \times 10^{-8} \mathrm{sec} .
$$

Indeed, evidence has already been given that $\Sigma^{+}$contamination is very small ${ }^{13}$ probably as a result of the very short lifetime of the $\Sigma^{+}$.

Again only events with origins in the $5 \mathrm{~cm}$ of producing material just above each chamber were considered. Because of their long mean life and consequent small detection probabilities, the mean detection probability for the $K^{+}$particles was computed from the more numerous sample of $\theta^{0} \mathrm{~s}$ on the assumption that their momentum distribution, at production, is the same as that of the $\theta^{0}$ 's, using the relation

$$
\bar{\pi}\left(K^{+}\right)=\sum_{N\left(\theta^{0}\right)} \frac{\pi\left(K^{+}\right)}{\pi\left(\theta^{0}\right)} / \sum_{N\left(\theta^{0}\right)} \frac{1}{\pi\left(\theta^{0}\right)} .
$$

In evaluating $N\left(K^{+}\right)$, only $K^{+}$particles with decay angles greater than $10^{\circ}$ were included and this fact was taken into account in the calculation of the $\pi\left(K^{+}\right)$ corresponding to each $\theta^{0}$. In addition it was required for each $K^{+}$included in the analysis that the detection probability of a $\theta^{0}$ with the same origin and momentum be greater than a certain minimum amount, and only $\theta^{0}$ s with detection probability greater than this amount were used.

\section{RESULTS OF THE ANALYSIS}

\section{A. Values of $I_{1}$ and $I_{2}$}

From an analysis of $60 \Lambda^{0}$ events, $54 \theta^{0}$ events, and $21 \Lambda^{0}-\theta^{0}$ pairs, the following values were obtained for $I_{1}$ and $I_{2}$ after suitable corrections were made for unseeable events emitted at large angles to the vertical.

$$
\begin{aligned}
& I_{1}=3.08_{-0.52}{ }^{+0.66}, \\
& I_{2}=1.86_{-0.31}{ }^{+0.40},
\end{aligned}
$$

where the quoted errors, which arise from the limited statistics and from random measurement errors, define a $50 \%$ confidence interval. 


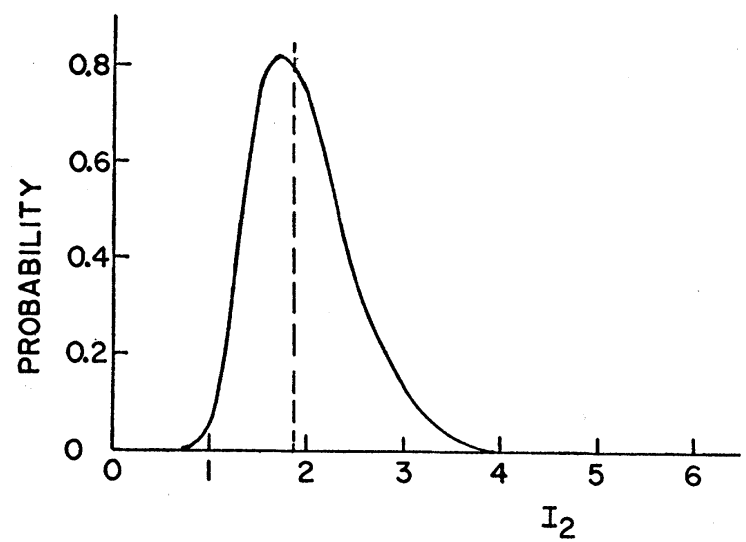

FIG. 3. Expected distribution of $I_{2}$ from a set of measurements of the same statistical accuracy as the present experiment. This curve is computed on the assumption that the true value of $I_{2}$ is the one obtained in the present experiment and shown in the figure by a dashed line.

Because of the small sizes of the numbers involved, a more detailed statistical analysis was carried out, in which a Monte Carlo calculation was used to evaluate the expected distribution of mean detection probabilities, and Poisson distributions for the numbers of events were folded in. The results of this analysis are shown in Figs. 2 and 3. From this calculation, the $98 \%$ confidence limits upon $I_{1}$ and $I_{2}$ were found to be $1.50<I_{1}<5.50$ and $1.00<I_{2}<3.50$.

In addition to the random errors just discussed, systematic effects can also have some importance. One such effect can arise if the $\Lambda^{0}$ or $\theta^{0}$ lifetimes used are in error. However, it is clear from Fig. 1 that the detection efficiency is rather insensitive to the lifetime except at very high momenta. Thus, the values of $I_{1}$ and $I_{2}$ are virtually independent of the lifetimes unless these are widely in error. Another source of systematic bias might arise if the scanning efficiency for doubles is not the same as for singles. Indeed, it is estimated that the scanning efficiency for single $V^{0}$ events is about $80 \%$, a result which might suggest a bias against the detection of pair events. However, every frame containing at least one $V^{0}$ decay has been thoroughly searched for others, in addition to which the probability of finding at least one decay in a picture where there are two is about $96 \%$. Hence, it is believed that scanning efficiency has introduced little bias.

\section{B. Value of $I_{3}$}

From an analysis of $58 \theta^{0}$ and $14 K^{+}$events, the following value for $I_{3}$ was obtained:

$$
I_{3}=3.85_{-0.75}+0.93 \text {, }
$$

where again the quoted errors are $50 \%$ confidence limits arising from all random effects.

Here, unlike the analysis for $I_{1}$ and $I_{2}$, the results are rather sensitive to the lifetimes used. The dependence on the $\theta^{0}$ lifetime arises from the fact that only the very lowest part of the $\theta^{0}$ momentum spectrum contributes appreciably to the predicted number of $K^{+}$particles, because of the long $K^{+}$lifetime. It is however, just this low-momentum part of the spectrum whose detection efficiency is most sensitive to the $\theta^{0}$ lifetime. Unfortunately the $\theta^{0}$ lifetime is not very well known, partly because of large statistical errors in determinations up to date, and partly because of the unknown effects introduced by a possible contamination of the anomalous $\theta^{0}$ events in the samples of $\theta^{0}$ s used. In view of recent work on anomalous $\theta^{0}$ events ${ }^{19}$ and recent determinations of the $\theta^{0}$ lifetime which give somewhat lower values than had been obtained previously, ${ }^{20}$ is seems likely that the lifetime value used in the present analysis may be, if anything, somewhat high. Consequently the value of $I_{3}$ was also computed for a $\theta^{0}$ lifetime of $6.5 \times 10^{-11} \mathrm{sec}$ with the following result,

$$
I_{3}=1.81_{-0.35}{ }^{+0.44} \text {. }
$$

The value of $I_{3}$ is also sensitive to the $K^{+}$lifetime, being proportional to it. However, the accuracy of the known value of this lifetime is about $10 \%$ so that the error introduced thereby into $I_{3}$ is not very important.

\section{DISCUSSION OF THE RESULTS}

\section{A. Estimates of $\varrho_{\theta}, \varrho_{\Lambda}$ and Production Probabilities}

If the assumptions leading to Eqs. (9a), (9b), and (10) are valid, then certain results follow immediately. In particular, it is clear that

$$
\begin{aligned}
\rho_{\theta} & >0.32_{-0.06}{ }^{+0.07}, \\
\rho_{\Lambda} & >0.54_{-0.10^{+0.11}} \\
P\left(\Lambda^{0} K^{+}\right) / P\left(\Lambda^{0} K^{0}\right) & <2.08_{-0.52}{ }^{+0.66}, \\
P\left(\Sigma^{ \pm} K^{0}\right) / P\left(\Lambda^{0} K^{0}\right) & <0.86_{-0.31}{ }^{+0.40} .
\end{aligned}
$$

It must be emphasized that the lower limits just quoted for $\rho_{\theta}$ and $\rho_{\Lambda}$ are not estimates of the actual values of these quantities unless $P\left(\Lambda^{0} K^{+}\right) / P\left(\Lambda^{0} K^{0}\right)$ and $P\left(\Sigma^{ \pm} K^{0}\right) / P\left(\Lambda^{0} K^{0}\right)$ are both negligible in comparison to unity. Although the available data are insufficient to give accurate experimental values for these ratios, they do indicate that both ratios are of the order of unity. It then follows that $\rho_{\theta}$ and $\rho_{\Lambda}$ are probably considerably larger than the lower limits given above. Indeed, in the approximation that protons and neutrons occur in equal numbers both in nuclei and in cosmic rays at sea level, and the $\pi^{+}$and $\pi^{-}$mesons are also equally numerous, one would expect that $P\left(\Lambda^{0} K^{+}\right) / P\left(\Lambda^{0} K^{0}\right)=1$. The corresponding value of $\rho_{\theta}$ would then be,

$$
\rho_{\theta}=0.65_{-0.11^{+0.13}} \text {. }
$$

${ }^{19}$ Kadyk, Trilling, Leighton, and Anderson, Bull Am. Phys. Soc. Ser. II, 1, 251 (1956).

${ }^{20} \mathrm{E}$. T. Booth, Proceedings of the Sixth Annual Rochester Conference on High-Energy Physics (Interscience Publishers, Inc., New York, to be published). 
From the same approximation it also follows that $P\left(\Sigma^{ \pm} K^{+}\right) / P\left(\Sigma^{ \pm} K^{0}\right)=1$, in which case one gets the following values for $\rho_{\theta}$ from the measurement of $I_{3}$ :

$$
\begin{array}{lll}
\rho_{\theta}=0.26_{-0.05}+0.06 & \text { if } & \tau_{\theta}=1.3 \times 10^{-10} \mathrm{sec}, \\
\rho_{\theta}=0.55_{-0.11}+0.13 & \text { if } & \tau_{\theta}=6.5 \times 10^{-11} \mathrm{sec} .
\end{array}
$$

The effects of not having equal numbers of neutrons and protons, and positive and negative $\pi$ mesons, while not in a completely clear direction, are probably not large enough to change the above estimates of $\rho_{\theta}$ very much.

\section{B. Effects of Processes Not Considered in the Previous Analysis}

The analysis just given has neglected the effects of all processes in which two $K$ particles are produced. These include the associations $K-\bar{K}$ and $\Xi-K-K$.

The effects of these processes can easily be discussed qualitatively by rewriting Eqs. (9) and (10) in the form

$$
\begin{aligned}
& I_{1}=\left(1 / \rho_{\theta}\right)(1+\lambda), \\
& I_{2}=\left(1 / \rho_{\Lambda}\right)(1+\xi), \\
& I_{3}=\left(1 / \rho_{\theta}\right) \eta,
\end{aligned}
$$

where $\lambda, \xi$, and $\eta$ are positive constants which in the approximations of the previous sections have the values

$$
\begin{aligned}
& \lambda_{0}=\frac{P\left(\Lambda^{0} K^{+}\right)}{P\left(\Lambda^{0} K^{0}\right)}, \quad \xi_{0}=\frac{P\left(\Sigma^{ \pm} K^{0}\right)}{P\left(\Lambda^{0} K^{0}\right)}, \\
& \eta_{0}=\frac{P\left(\Lambda^{0} K^{+}\right)+P\left(\Sigma^{ \pm} K^{+}\right)}{P\left(\Lambda^{0} K^{0}\right)+P\left(\Sigma^{ \pm} K^{0}\right)} .
\end{aligned}
$$

It can easily be seen that the effects of the processes neglected are to change the values of $\lambda, \xi$, and $\eta$ to different but still positive values. Hence, the lower limits on $\rho_{\theta}$ and $\rho_{\Lambda}$ obtained previously are still valid.

The effects of the neglected processes on $\lambda, \xi$, and $\eta$ can be summarized in the following way:

$$
\lambda<\lambda_{0}, \quad \xi>\xi_{0}, \quad \eta<\eta_{0} \quad \text { or } \quad \eta>\eta_{0} .
$$

Information on the extent to which the values of $\lambda, \xi$, and $\eta$ are changed can be obtained by a study of the numbers of $\theta^{0}-\theta^{0}$ pairs, $\Xi^{-}$events and $K^{-}$events, all of which can arise only in the neglected processes. In view of the relative scarcity of all these types of events, it is believed that the true values of $\lambda, \xi$, and $\eta$ are not significantly different from $\lambda_{0}, \xi_{0}$, and $\eta_{0}$.

\section{Effects Due to Anomalous $\theta^{09} \mathrm{~s}$}

A fundamental assumption, which is satisfied only to an approximate degree, has been made in the foregoing analysis to obtain estimates of $\rho_{\theta}$ and $\rho_{\Lambda}$, namely that all events identified as $\theta^{\circ}$ 's by the criteria of Table I are examples of the decay,

$$
\theta^{0} \rightarrow \pi^{+}+\pi^{-}, \quad \tau \approx 10^{-10} \mathrm{sec} .
$$

It is now well known that there are "anomalous" events which, while they may fulfill one or more of the $\theta^{0}$ identification criteria, do not have the decay scheme above. In view of the fact that such events have been excluded from this analysis only when clear evidence of their being anomalous existed, it is very likely that some did get mixed in with normal $\theta^{0}$ 's. Too little is known about these anomalous events at the present time to be able to say to what extent their effect is important, although the fact that only a very small number of these decays has been found would tend to suggest that they do not introduce important changes. In any case, however, if the anomalous events are neutral $K$-particle decays, their presence will tend to make the values of $I_{1}$ and $I_{3}$ somewhat too low, and the corresponding estimates of $\rho_{\theta}$ too high.

A somewhat different interpretation of the analysis given in this paper can be got by redefining the quantities $\rho_{\theta}$ and $\rho_{\Lambda}$ in the following way. Let $\rho_{\theta}{ }^{\prime}$ and $\rho_{\Lambda}{ }^{\prime}$ be the fractions of $K^{0}$ particles and $\Lambda^{0}$ particles, respectively, which decay into charged secondaries, regardless of decay scheme or lifetime. It can be seen from Fig. 1 that the lifetime which maximizes the detection probability is just about equal to the ordinary $\Lambda^{0}$ or $\theta^{0}$ lifetime. It then follows from the fact that all observed decays have been assumed to be one of the well-known types that the detection probabilities have, if anything, been overestimated. Hence, the estimates of $\rho_{\theta}$ and $\rho_{\Lambda}$ obtained above are lower limits to the values of $\rho_{\theta}{ }^{\prime}$ and $\rho_{\Lambda}^{\prime}$.

\section{INTERPRETATION AND CONCLUSIONS}

The data and analysis which have been presented in the previous sections have shown that both from the standpoint of the identities of individual events and from the numbers involved, associated production of strange particles is strongly indicated. The data have also yielded rough estimates of the values of $\rho_{\theta}$ and $\rho_{\Lambda}$. These are subject to rather large errors and uncertainties; therefore, a detailed interpretation of them is not justified. It is, however, of some importance to note to what extent the interpretation of these values depends upon present knowledge of the production of strange particles. Thus, the value of $\rho_{\theta}$ given by the value of $I_{1}$ must really be interpreted as the fraction of all neutral $K$ particles, produced in association with observed $\Lambda^{0} s$, which decay by the usual $\theta^{0}$ scheme. Similarly the value of $\rho_{\Lambda}$ given by $I_{2}$ is really the fraction of neutral hyperons of strangeness -1 , which decay by the usual $\Lambda^{0}$ mode, produced in association with observed $\theta^{0} s$. On the other hand, the value of $\rho_{\theta}$ derived from $I_{3}$ is more in line with the definition originally given, that is, 
the fraction of neutral $K$ particles, produced in any way, which decay by the $\theta^{0}$ scheme. Thus, although statistics and lifetime uncertainties do not permit one to say that the values of $\rho_{\theta}$ obtained from $I_{1}$ and $I_{3}$ disagree, the fact that the latter appears somewhat lower could conceivably arise from a real physical effect.

It appears clear that further elucidation of these questions will have to be carried out under the much more highly controllable conditions available with highenergy accelerators.

\section{ACKNOWLEDGMENTS}

The authors wish to express their appreciation to Professor C. D. Anderson for valuable discussions, and to G. Neugebauer and R. Luttermoser for assistance in the scanning and analysis of the data.

PHYSICAL REVIEW

VOLUME 104, N UMBER 6

D E C E M B E R 15,1956

\title{
Momentum of Nucleons in Various Nuclei from the High-Energy Photoeffect*
}

A. Wattenberg, A. C. Odian, P. C. Stein, AND H. Wilson, Physics Department and Laboratory for Nuclear Science, Massachusetts Institute of Technology, Cambridge, Massachusetts

AND

R. WeINstein, $\dagger$ Brandeis University, Waltham, Massachusetts

(Received August 6, 1956)

\begin{abstract}
The previously reported studies of the high-energy photoejection of neutrons and protons showed the basic validity of the "quasi-deuteron" model. Due to the motion of neutrons and protons in a complex nucleus one observes that the kinematics of the "quasi-deuteron" disintegration are modified from those of the photodisintegration of a free deuteron at rest. The modification of the kinematics was measured for $\mathrm{Li}, \mathrm{C}, \mathrm{O}, \mathrm{Al}$, and $\mathrm{Cu}$ in an attempt to compare the "average" momentum of the nucleons in these nuclei. It is found that a Fermi momentum distribution (with $T=0^{\circ}$ ) does not fit the observed data. A calculation employing only conservation of momentum and assuming a three-dimensional Gaussian momentum distribution for the neutrons and protons, gives an expression that fits the data quite well for $\mathrm{Li}, \mathrm{C}$, and $\mathrm{O}$. The $1 / e$ values of the Gaussians for $\mathrm{Li}, \mathrm{C}$, and $\mathrm{O}$, respectively are 8,19 , and $19 \mathrm{Mev}$. These values have been corrected for the experimental angular resolution and for refraction at the nuclear surface.
\end{abstract}

\section{INTRODUCTION}

W HEN nuclei are bombarded by high-energy $\mathrm{x}$-rays, neutrons and protons are observed in coincidence, ${ }^{1}$ which is in agreement with the qualitative prediction of the "quasi-deuteron" model of Levinger." On this model, high-energy x-rays interact with a pair of nucleons in a complex nucleus rather than with the entire nucleus.

A previous paper by the authors ${ }^{3}$ on the photoejection of high-energy neutrons and protons gives some of the results of a series of experiments undertaken at the M.I.T. Synchrotron. The experiments previously reported indicate that the mechanisms involved in a complex nucleus, in the simultaneous photoejection of a neutron and proton, are the same mechanisms responsible for the photodisintegration of a free deuteron.

The kinematical relationships for a free deuteron (at rest) are modified in a complex nucleus by the initial

* This work has been supported in part by the joint program of the Office of Naval Research and the U. S. Atomic Energy Commission.

$\dagger$ Now at the Massachusetts Institute of Technology, Cambridge, Massachusetts.

1 M. Q. Barton and J. H. Smith, Phys. Rev. 95, 573 (1954);

Meyers, Odian, Stein, and Wattenberg, Phys. Rev. 95, 576 (1954).

2 J. S. Levinger, Phys. Rev. 84, 43 (1951).

3 Odian, Stein, Wattenberg, Feld, and Weinstein, Phys. Rev. 102, 837 (1956). momentum of the center of mass of the neutron-proton pair. Specifically, if the protons energy and angle are held fixed, the coincident neutrons are observed to have an angular spread about the angle at which one observes the neutrons from deuterium. In the preliminary measurements, the spread in neutron angles was found to be in semiquantitative agreement with what one would expect if the spread arose from the initial momentum of the center of mass of the neutron-proton pairs. Therefore, as part of the series of measurements, we undertook to study the momentum of nucleons in various nuclei; this article is a report on these measurements.

At the time these momentum studies were undertaken, the published data on the momentum of nucleons was somewhat discrepant and was limited essentially to data on $\mathrm{Li}, \mathrm{C}$, and $\mathrm{O}$. The data available at that time are listed in Table I. More recently, Wilcox and Moyer ${ }^{4}$ have used the quasi-elastic scattering of protons to study the momentum in $\mathrm{Li}, \mathrm{Be}$, and $\mathrm{B}$, and Selove ${ }^{5}$ has used the deuteron pickup process to study Be and $\mathrm{C}$.

It is to be pointed out that the earliest experiments and the meson experiments were in general not motivated by a desire to determine momentum distribution,

${ }^{4}$ J. M. Wilcox and B. J. Moyer, Phys. Rev. 99, 875 (1955). ${ }^{5}$ W. Selove, Phys. Rev. 101, 231 (1956). 